\title{
Measuring Stellar Radial Velocity using Markov Chain Monte Carlo(MCMC) Method
}

\author{
Yihan Song $^{1}$, Ali Luo ${ }^{2 *}$ and Yongheng Zhao $^{3}$ \\ ${ }_{1,2,3}$ National Astronomical Observatories, Chinese Academy of Sciences, \\ 20A Datun Road, Chaoyang District, Beijing, China \\ email: yhsong, lal, yzhao@lamost.org
}

\begin{abstract}
Stellar radial velocity is estimated by using template fitting and Markov Chain Monte Carlo(MCMC) methods. This method works on the LAMOST stellar spectra. The MCMC simulation generates a probability distribution of the RV. The RV error can also computed from distribution.
\end{abstract}

Keywords. Radial Velocity, Templates, MCMC, etc.

Radial Velocity is a very important parameter for stars. It can be measured on stellar spectra. Two methods are used normally. One is measuring the wavelength of the emission or absorption lines. The other one is fitting the spectrum with model templates. Lines measuring is suit for spectra with high resolution and accurate calibration. For the low resolution spectra, such as SDSS and LAMOST spectra, templates fitting is more suitable. However, the goodness of fitting is a problem.

The method is designed for computing the radial velocity for LAMOST spectra. The resolution of LAMOST spectra is 2000. Templates fitting is introduced in this method. Kurucz models and Hammer templates compose the templates. The fitting method is cross-correlation. The first step of this method is to find the best model from templates. The second step is using the MCMC to compute the radial velocity. We use the correlation value as the probability to adjust the RV. For every spectra, we make 1000 times simulation to build the probability distribution.
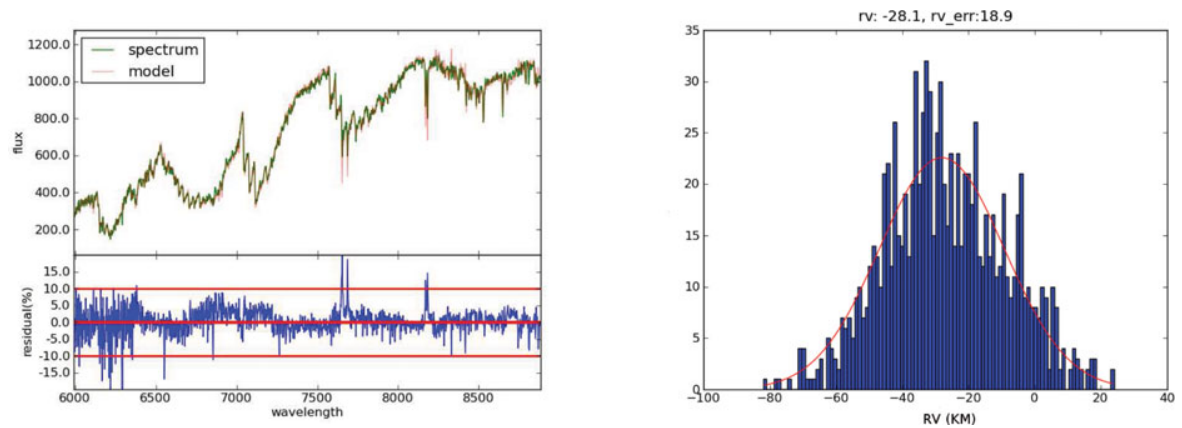

The left figure includes a M star from LAMOST (top, green) and its best model(top, red) and the residual (bottom). The right figure shows the MCMC probability distribution (blue, histogram) and its fitted curve (red, Gaussian function). From the center of the fitted Gaussian curve, we can output the radial velocity. The error of the RV is the corresponding $\sigma$. 